\title{
SELF-REGULATION PROCESSES IN LEARNER-GENERATED DIGITAL MEDIA (LGDM) ASSIGNMENTS
}

\author{
J. Reyna, P. Meier \\ University of Technology Sydney (AUSTRALIA) \\ jorge.reyna@uts.edu.au
}

\begin{abstract}
Digital Media as an assessment tool is used in Higher Education as a vehicle to learn the subject content and to develop communication skills. Although it became a common practice in the last decade, the research field is considered to be under-theorised, under-researched and barely sufficient. Several gaps in the literature have been identified such as a practical model to implement digital media in the classroom, lack of educator's know-how in digital media production, and effective marking rubrics. Additionally, methodological approaches to measure LGDM effectiveness in the classroom as a learning tool are in embryonic stages. The literature reported studies with small sample size, qualitative approaches and lack of student training and support. An extra layer of complexity is the different digital media types such as audio podcast, digital story, animation, and video. These digital artefacts require a different set of production skills. Therefore, the comparison between studies can be problematic. However, many advantages of using LGDM has been highlighted by researchers with questionable empiric evidence. Due to the nature of digital media production workflow, characterised by being time-consuming, iterative and resource-intensive, we posit that students engaging in LGDM assignments require self-regulation skills to succeed on the task. For instance, developing a storyboard that requires to be evidence-based, visualisation of the content using multimodality, learning the different applications to produce the assets for the project, and engaging in the final production phase will require self-regulation skills. This paper discusses how understanding selfregulation processes in LGDM assignments can lead to a personalisation of student learning experience when LGDM assignments are used in the classroom.
\end{abstract}

Keywords: Learner-generated digital media, digital media assignments, self-regulation with digital media, user-generated content, authentic assessments.

This is a preview of the paper to be published at INTED 2019 Proceedings at the end of March 2019.

\section{Reference:}

Reyna, J \& Meier, P (2019). Self-Regulation Processes in Learner-Generated Digital Media (LGDM) Assignments. Exploring New Frontiers in Education. The 13th annual International Technology, Education and Development Conference, INTED2019, Valencia (Spain), March 11th-13th. 